\title{
Indifferent streptococci in normal and purulent eyes of neonates
}

\author{
JC REEDER, AJ WESTWELL, DN HUTCHINSON
}

From the District Laboratory, Preston Infirmary, Preston

SUMMARY The incidence of indifferent streptococci in the eyes of neonates less than 6 days old was investigated. The isolation of indifferent streptococci was significantly higher in infants with sticky eyes. Speciation using a shortened identification scheme was carried out on isolates; Streptococcus mitior and Streptococcus sanguis were the most common species in both purulent and non-infected eyes. Streptococcus mutans, a species not normally found in edentulous infants, comprised $14 \%$ of indifferent streptoccoi from neonates with sticky eyes but only $1 \%$ of those from infants with non-purulent eyes. It is suggested that the role of indifferent streptococci in neonatal conjunctivitis will be resolved only if speciation is performed.

The incidence of neonatal conjunctivitis in the United Kingdom has been reported to range from $2.6 \%$ to $12 \% .^{1-3}$ Neisseria gonorrhoea has long been acknowledged as a cause of ophthalmia neonatorum, but Staphylococcus aureus is now the pathogen most frequently isolated from infants with a sticky eye. Infections due to Chlamydia trachomatis usually produce a characteristic clinical picture and, unless there has been rupture of the fetal membranes several days before birth, seldom manifest themselves until the infant is 5 days old. Also associated with premature rupture of the membranes are infections of the eye due to Mycoplasma hominis. ${ }^{4}$

Several investigations have shown that the viridans streptococci can be isolated more often from infants with ophthalmia neonatorum than from control babies. ${ }^{356}$ The higher rate of isolation of the viridans streptococci from infected eyes raises the possibility that this organism may play a part in the pathogenesis of sticky eye. The isolates have been widely designated Streptococcus viridans, the name being used as a convenient but often inappropriate label; we prefer the term indifferent streptococci because it includes species that do not exhibit the typical green $\alpha$ haemolysis on chocolate agar. The development of schemes by Parker and Ball ${ }^{7}$ and Facklam ${ }^{8}$ and the introduction of a useful shortened scheme for identifying the indifferent streptococci" followed by the production of a commercial kit $^{10}$ have all made it possible to speciate almost all isolates. The following studies were initiated to identify the species of indifferent streptococci isolated from infected and non-infected eyes of neonates and to Accepted for publication 24 April 1985 determine whether the increased isolation from infected eyes was associated with one or more species.

\section{Material and methods}

\section{STUDY A}

Subjects

All infants born in the maternity unit at this hospital over two periods of eight weeks were studied. Brief clinical details of the pregnancy and the method of delivery were recorded.

\section{Specimens}

Conjunctival swabs from both eyes were collected by the same operator between the third and fifth days of life. The infants' eyes were examined and the presence of inflammation or discharge noted before swabbing, which specifically took place before the mother or nurse washed the child in the morning. To avoid contamination specimens from purulent eyes were collected after those from non-infected eyes. The swabs, tipped with cotton wool, were placed in Stuart's charcoal transport medium," transported immediately to the laboratory, and cultured within one hour.

Each swab was inoculated on to two plates of $5 \%$ blood agar and one plate of chocolate agar (Oxoid Columbia agar base with Difco defibrinated blood). One blood agar plate was incubated aerobically and one anaerobically in $90 \%$ hydrogen and $10 \%$ carbon dioxide, both at $37^{\circ} \mathrm{C}$. The chocolate agar plate was incubated in a mixture of $95 \%$ air and $5 \%$ carbon dioxide in a humid environment at $37^{\circ} \mathrm{C}$. All plates were examined after 24 and 48 hours. A 
smear was prepared from each swab and stained by Preston and Morrell's modification of Gram stain. ${ }^{12}$ Only those with pus cells were regarded as potentially infected.

\section{Identification of isolates}

Control organisms comprised a range of streptococci-that is, 10 type strains from the National Collection of Type Cultures and six recent clinical isolates. Representatives from each colony type that had been isolated from the infants' eyes were subcultured for identification. Organisms other than those seeming to be indifferent streptococci were identified by the methods of Cowan and Steel. ${ }^{13}$

Organisms suspected of belonging to the indifferent streptococci on the basis of colonial morphology, $\alpha$ haemolysis, or non-haemolysis, negative catalase reaction, and Gram stained smear were stored on blood agar slopes at $4^{\circ} \mathrm{C}$. Isolates were examined in batches. When required for identification the organisms were subcultured on to blood agar plates and incubated overnight at $37^{\circ} \mathrm{C}$ in $5 \%$ carbon dioxide. A single colony was then picked into a $5 \mathrm{ml}$ Todd Hewitt broth, which was incubated in similar atmospheric conditions with the cap loosened. The media for identification were inoculated from this overnight culture.

The shortened scheme of Waitkins et al was used to identify indifferent streptococci." The following tests were used: fermentation of lactose, raffinose, arabinose, sorbitol, mannitol, and pyruvate; salt tolerance $(6.5 \%)$; growth on $10 \%$ and $40 \%$ bile salt concentrations; hydrolysis of starch and aesculin; presence of arginine dehydrolase; Voges-Proskaur test; detection of the extracellular polysaccharides dextran and laevan; and survival at $60^{\circ} \mathrm{C}$ for 30 minutes. Most strains were also examined by the API 20S streptococci identification kit (API Laboratory Products Ltd).

\section{STUDY B}

Routine isolations of indifferent streptococci

Over an additional eight months all isolations of indifferent streptococci that had been made from conjunctival swabs collected from infants less than six days old with sticky eyes were stored and identified as described previously.

\section{Results}

STUDY A

Conjunctival swabs were collected from 405 infants ( 154 from the first eight weeks, 251 from the second eight weeks). For analysis the infants' two eyes were regarded as one-that is, a child with one or both eyes affected was included in the conjunctivitis group only. The proportion of infants with sticky eyes in the two periods of eight weeks was 22 $(14.3 \%)$ and $39(15.5 \%)$, respectively, with an overall incidence of $15.1 \%$. Eight infants were recorded clinically as having sticky eyes, but as pus cells were not detected in the Gram film they were included in the non-infected group.

Fifty nine babies had been delivered by caesarean section (four of these developed eye infections) and 57 of the 346 infants delivered vaginally showed signs of infection.

Table 1 shows a comparison of the species of organisms isolated from both non-infected and infected eyes. Coagulase negative staphylococci were isolated most commonly in both infected and non-infected eyes. Indifferent streptococci were the next most common organisms in both groups but were significantly increased $(p<0.001)$ in those infants with sticky eyes. Similarly, the isolation of Staph aureus from this second group was, at $16.4 \%$, much higher than the $1.5 \%$ rate of isolation from non-infected infants. Branhamella catarrhalis was isolated from a large proportion of infants in both groups. Bacteria were not isolated from the eyes of four infants with purulent discharge (none of them had had antibiotics instilled before swabs were collected).

\section{Speciation of streptococci isolates}

One hundred and one of the 102 strains isolated from non-infected eyes were available for identification tests. The 38 strains isolated from infected eyes (study A) and an additional 63 strains collected routinely from the eyes of infants with neonatal conjunctivitis (study B) were examined. Table 2 shows the number and proportion within each species. The species were designated according to the recommendations of Parker and Ball. ${ }^{7}$

The dextran negative Strep mitior and Strep sanguis were the most commonly isolated species in both groups of infants. Strains of dextran negative Strep mitior were recovered significantly more frequently from infants with sticky eyes. Strep sanguis was less commonly isolated. Strep mutans was isolated from 14 sticky eyes, but only one isolation was made from the non-infected group. This was from an infant who was clinically regarded as having a sticky eye, but pus cells were not seen in the Gram film.

\section{Agreement between API 20 S streptococcal kit and short identification scheme}

In only 10 of 193 comparisons made between the two systems were there discrepancies in the speciation. Eight strains coded as Strep sanguis by the API 
Table 1 Isolates from conjunctival swabs of neonates (figures are numbers (\%))

\begin{tabular}{|c|c|c|}
\hline Organism & Non-infected eyes $(n=344)$ & Sticky eyes $(n=61)$ \\
\hline $\begin{array}{l}\text { Coagulase negative staphylococci } \\
\text { Indifferent streptococci } \\
\text { Staphylococcus aureus } \\
\text { Branhamella catarrhalis } \\
\text { Escherichia coli } \\
\text { Proteus mirabilis } \\
\text { Haemolytic streptococcus group B } \\
\text { Klebsiella sp } \\
\text { No growth }\end{array}$ & $\begin{aligned} 275 & (79 \cdot 9) \\
102 & (29 \cdot 7) \\
5 & (1 \cdot 5) \\
69 & (20 \cdot 0) \\
1 & (0 \cdot 3) \\
1 & (0 \cdot 3) \\
1 & (0 \cdot 3) \\
38 & (11 \cdot 0)\end{aligned}$ & $\begin{array}{l}41(67.2)(p=<0.05) \\
38(62.3)(p=<0.001) \\
10(16.4) \\
9(14.8)(p=<0.5) \\
3(4.9) \\
1(1.6) \\
1(1.6) \\
4(6.6)\end{array}$ \\
\hline
\end{tabular}

Table 2 Species of indifferent streptococci isolated from healthy and sticky eyes of neonates (figures are numbers (\%))

\begin{tabular}{lcc}
\hline Organism & Non-infected eyes $(n=101)$ & Sticky eyes $(n=101)$ \\
\hline Streptococcus mitior dextran negative & $45(44 \cdot 6)$ & $64(63 \cdot 4)(\mathrm{p}=<0 \cdot 01)$ \\
Streptococcus sanguis & $36(35 \cdot 6)$ & $19(18 \cdot 8)(\mathrm{p}=<0 \cdot 01)$ \\
Streptococcus mitior dextran positive & $10(9 \cdot 9)$ & $1(0 \cdot 99)$ \\
Streptococcus faecalis & $4(3 \cdot 96)$ & $1(0 \cdot 99)$ \\
Streptococcus bovis I & $1(0 \cdot 99)$ & $2(1 \cdot 98)$ \\
Streptococcus bovis II & $1(0 \cdot 99)$ & $14(13 \cdot 9)$ \\
Streptococcus milleri & $1(0 \cdot 99)$ & $1(0 \cdot 99)$ \\
Streptococcus equinus & $1(0 \cdot 99)$ & $1(0 \cdot 99)$ \\
Streptococcus mutans & & \\
Streptococcus salivarius & & \\
\hline
\end{tabular}

20S streptococci kit were classified as Strep mitior by the method of Waitkins et al and two strains that were coded Strep mitior by the API 20S streptococci scheme were speciated Strep sanguis by the shortened identification scheme. In each case the discrepancy was not in the biochemical test results but in the different classification schemes applied-that is, Facklam $^{8}$ (API 20S streptococci) and Parker and Ball' (shortened scheme).

\section{Discussion}

The isolation of indifferent streptococci from 38 sticky eyes compared with 102 non-infected eyes was significant $(p<0.001)$. The rate of isolation for both groups of infants in this study was much greater than that reported by others, who have also remarked on the significantly higher incidence of viridans streptococci in the infected eyes of neonates. $^{356}$ Factors that could have accounted for our high rate of isolation were the active pursuit of streptococci and the inclusion of non-haemolytic strains to the $\alpha$ haemolytic isolations.

Strep mitior was the most commonly identified species in both infected and non-infected eyes, an observation also recorded by Bone. ${ }^{14}$ The significant increase in the proportion of dextran negative Strep mitior from infected eyes suggests that this organism may have a pathogenic role, whereas the reduction of Strep sanguis would indicate that it was present as a commensal.

The isolation of Strep mutans from clinically infected eyes alone suggests that it may have a pathogenic role. It is generally accepted that Strep mutans cannot be isolated from the oral cavity of edentulous infants, ${ }^{1516}$ and its isolation in this study may reflect its presence in the oral flora of the mother and the child's attendants. Apart from its association with dental caries, ${ }^{17}$ it has been regularly isolated from patients with endocarditis. ${ }^{7}$ Strep mutans produces dextran, a factor that is thought to increase the adherence of the bacterium to damaged heart valves. ${ }^{18}$ Similarly, in neonatal conjunctivitis production of dextran could be advantageous for certain streptococcal species, but a reduction in the rate of isolation from infected eyes of other species that produce dextran (Strep sanguis and dextran positive Strep mitior) suggests that this factor has no appreciable affect on indifferent streptococci.

Staph epidermidis was the organism most commonly isolated from both non-infected and sticky eyes, and though there seems to be a significant difference between the rates of isolation in the two groups, this is probably explained by the difficulty of obtaining a good specimen. Owing to the area of the swabbing site and the unpredictable nature of the patient, many of the swabs would have been contaminated by the flora of the skin around the eye socket. When frank pus is present it is easier to obtain satisfactory material, and this factor alone may account for the discrepancy between the two groups.

There seems to be an association of Staph aureus and the Enterobacteriaceae with clinical infection, although the numbers are too small for statistical analysis. Nevertheless, the isolation of these organ- 
isms from non-infected eyes is a reminder that they may have a commensal as well as a pathogenic role. ${ }^{3}$ $B$ catarrhalis was present in a large proportion of swabs from both groups, though there was no significant difference between the rate of isolation.

The incidence of neonatal conjunctivitis in this study was $15.5 \%$, which is similar to the $12 \%$ reported by Pierce et al ${ }^{3}$, who excluded those infants in whom anatomical abnormalities prevented a resolution of their discharge..$^{14}$ As our follow up was restricted all infants with pus cells in the discharge were included.

Acquisition of a bacterial flora in neonates starts immediately after birth. Rotimi et al isolated upper respiratory commensal organisms from the oral flora of infants 24 hours old. ${ }^{20}$ The proportion of infants colonised by the indifferent streptococci increased rapidly after birth until by the sixth day of life these organisms could be recovered from the oral cavity of almost all the neonates studied. Likewise, Prentice et al found that organisms were more commonly isolated from eye swabs collected from infants aged 3-6 days that from neonates $1-2$ days old. ${ }^{5}$ The high overall rate of isolation found in this study reflects the age of the infants (3-5 days) at the time the eye swabs were collected and the difficulties associated with collection.

As we did not examine the eyes for the presence of chlamydia, urea plasma, other mycoplasma, viruses, and anaerobic bacteria it is possible that the changes in the proportion of respective indifferent streptococcal flora may reflect altered growth conditions created by the presence of one of these agents. We would agree with Bone ${ }^{14}$ that the list of potential pathogens in neonatal conjunctivitis should include most conjunctival commensals but would suggest that speciation of indifferent streptococci may clarify the role of this group of organisms in the pathogenesis of neonatal conjunctivitis.

We thank Dr Sheena Waitkins for helpful discussions, the paediatric, obstetric, and maternity staff of the Preston Health District for their cooperation, and Mrs M Collinson for typing the manuscript.
References

' Hurley R. Epidemic conjunctivitis in the newborn associated with coagulase negative negative staphylococci. Journal of Obstetrics and Gynaecology of the British Commonwealth 1966;73:990-2.

${ }^{2}$ Watson PG, Gairdner D. TRIC agent as a cause of neonatal eye sepsis. Br Med J 1968;iii:527-8.

${ }^{3}$ Pierce JM, Ward ME, Seal DV. Ophthalmia neonatorum in the 1980 's: incidence, aetiology and treatment. $\mathrm{Br} J$ Ophthalmol 1982; 66: 728-31.

${ }^{4}$ Jones DM. Tobin B. Neonatal eye infections due to Mycoplasma hominis. Br Med J 1968;iii:467-8.

s Prentice MJ, Hutchinson GR, Taylor-Robinson D. A microbiological study of neonatal conjunctivae and conjunctivitis. Br J Ophthalmol 1977;61:601-7.

${ }^{\circ}$ McGill RET. The sticky eyed infant. Br Med J 1983;287:428.

' Parker MT, Ball LC. Streptococci and aerococci associated with systemic infection in man. J Med Microbiol 1976;9:275-302.

${ }^{8}$ Facklam RR. Physiological differentiation of viridans streptocci. J Clin Microbiol 1977;5:184-201.

${ }^{4}$ Waitkins SA, Ball LC, Fraser CAM. A shortened scheme for the identification of indifferent streptococci. J Clin Pathol 1980;33:47-52.

${ }^{10}$ Waitkins SA, Ball LC, Fraser CAM. Use of the API-ZYM system in rapid identification of haemolytic and non-haemolytic streptococci. J Clin Pathol 1980;33:53-7.

"Stuart RD. Transport medium for specimens in Public Health bacteriology. Public Health Reports (Washington) 1959;74:431-8.

12 Preston NW, Morrell A. Reproducible results with the Gram stain. J Pathol Bacteriol 1962:84:241.

${ }^{13}$ Cowan ST, Steel KG. Manual for the identification of medical bacteria. London: Cambridge University Press, 1974.

${ }^{14}$ Bone FJ. The sticky eyed infant. Br Med J 1983;286: 2060.

is Catalanotto FA, Shklair IL, Keene HJ. Prevalence and localisation of Streptococcus mutans in infants and children. J Am Dent Assoc 1975;91:606-9.

${ }^{16}$ Berkowitz RJ, Jordan HV, White G. The early establishment of Streptococcus mutans in the mouths of infants. Arch Oral Biol 1975;20: 171-4.

17 Hamada S, Slade HD. Biology immunology and cariogenicity of Streptococcus mutans. Microbiol Rev 1980;44:331-84.

${ }^{18}$ Pellitier LL, Coyle M, Petersdors R. Dextran production as a possible virulence factor in streptococcal endocarditis. Proc Soc Exp Biol Med 1978;158:415-20.

14 Taylor D. The sticky eyed infant. Br Med J 1983;286:1770-1.

${ }^{20}$ Rotimi VO, Duerden BI. The development of the bacterial flora in normal neonates. J Med Microbiol 1981;14:51-62.

Requests for reprints to: Dr DN Hutchinson, District Laboratory, Preston Infirmary, Meadow Street, Preston, Lancs PR1 6PS, England. 